\title{
Effects of dezocine for the prevention of postoperative catheter-related bladder discomfort: a prospective randomized trial
}

This article was published in the following Dove Press journal:

Drug Design, Development and Therapy

\author{
Guang-Fen Zhang ${ }^{1} *$ \\ Jie Guo ${ }^{2, *}$ \\ Li-Li Qiu' \\ Shu-Ming $\mathrm{Li}^{1}$ \\ Man Zheng ${ }^{2}$ \\ Jiang-Yan Xia' \\ Jian-Jun Yang ${ }^{1,3}$
}

'Department of Anesthesiology, Zhongda Hospital, School of Medicine, Southeast

University, Nanjing, Jiangsu, People's Republic of China; ${ }^{2}$ Department of Anesthesiology, Affiliated Hospital of Nanjing University of Traditional Chinese Medicine, Nanjing, Jiangsu, People's Republic of China; ${ }^{3}$ Department of Anesthesiology, The First Affiliated Hospital of Zhengzhou University, Zhengzhou, Henan, People's Republic of China

*These authors contributed equally to this work

Correspondence: Jian-Jun Yang Department of Anesthesiology, Zhongda Hospital, School of Medicine, Southeast University, No.87 Dingiaqiao road, Nanjing 210009, People's Republic of China

Tel +86037166913114

Email yjyangji@I26.com
Purpose: To evaluate the effects of dezocine on the prevention of postoperative catheterrelated bladder discomfort (CRBD).

Patients and methods: Ninety-six adult patients undergoing abdominal surgery with urinary catheterization under general anesthesia were randomized into dezocine and control (flurbiprofen) groups. The postoperative CRBD, pain score, sedation score and adverse effects were evaluated at $0,1,2$ and 6 hrs after tracheal extubation.

Results: The primary outcome showed a lower incidence of CRBD at $1 \mathrm{hr}$ post-extubation in the dezocine group (29.17\%) than the control group $(58.33 \%, P<0.01)$. The incidences at 0 and $2 \mathrm{hrs}$ post-extubation and the overall incidence were also lower in the dezocine group than the control group (all $P<0.05$ ). The severity of CRBD at $0,1,2$ and $6 \mathrm{hrs}$ and the pain, sedation score and other adverse effects were comparable between the two groups $(P>0.05)$; however, the overall severity of CRBD was decreased in the dezocine group compared with the control group $(P<0.05)$.

Conclusion: Intraoperative dezocine reduces the incidence and severity of postoperative CRBD without clinically relevant adverse effects.

Keywords: dezocine, catheter-related bladder discomfort, general anesthesia, postoperation

\section{Introduction}

Catheter-related bladder discomfort (CRBD) is a clinical syndrome described as an urge to pass urine or as discomfort in the suprapubic region due to stimulation by the urinary catheter during recovery from general anesthesia. ${ }^{1}$ The incidence of CRBD ranged from $47 \%$ to $95 \%$ during the postoperative period in patients with urinary catheterization. $^{2-5} \mathrm{CRBD}$ is extremely distressing to patients and usually accompanied by behavioral responses including strong vocal responses, flailing limbs and attempting to pull out the urinary catheter. ${ }^{2}$ Moreover, CRBD increases postoperative pain and agitation. $^{6-8}$ Therefore, attention and early intervention are needed for these patients.

Involuntary contractions of the bladder muscle triggered by muscarinic receptors are involved in the pathogenesis of CRBD, thus muscarinic antagonists including butylscopolamine, solifenacin, darifenacin, oxybutynin, glycopyrrolate, and tolterodine can improve CRBD symptoms. ${ }^{9-13}$ Moreover, drugs with other mechanisms, including anesthetics (ketamine, tramadol, dexmedetomidine and lidocaineprilocaine cream), antiepileptics (gabapentin and pregabalin) and other drugs (amikacin, paracetamol and resiniferatoxin) have been reported to be effective in CRBD prevention. ${ }^{14-23}$ In addition to pharmaceutical therapies, other approaches have 
been successfully used to improve CRBD, eg, caudal block and dorsal penile nerve block. ${ }^{24}$

Dezocine is a mixed-opioid agonist/antagonist and often used for perioperative pain management. ${ }^{25-29}$ In clinical practice, we found that patients receiving dezocine for the treatment of postoperative pain appeared to suffer from less CRBD. However, the effect of dezocine on the prevention of CRBD has not been reported. Additionally, the spinal effect of dezocine through interactions with $\kappa$-receptors can produce a unique action in the treatment of visceral pain. ${ }^{26-29}$ Therefore, we hypothesized that dezocine is beneficial for $\mathrm{CRBD}$ and designed a prospective randomized trial to evaluate the effects of dezocine on the prevention of CRBD in patients undergoing abdominal surgery by investigating the incidence and severity of CRBD within 6 hrs after tracheal extubation.

\section{Materials and methods}

\section{Patients}

This study was conducted in accordance with the Declaration of Helsinki and reported in line with the Consolidated Standards of Reporting Trials (CONSORT) Guidelines. After receiving approval from the Institutional Ethics Committee for Clinical Research of Zhongda Hospital, Affiliated to Southeast University (approval no.: 2017ZDSYLL044-P01; August 18, 2017) and written informed consents from all patients, this prospective, randomized, and parallel design trial was performed. The protocol for this clinical trial was registered at ClinicalTrials.gov (registration no.: NCT03147066; May 10, 2017). Patients aged 18-65 years with American Society of Anesthesiologists (ASA) Physical Status I or II and scheduled for elective abdominal surgery with urinary catheterization for at least $6 \mathrm{hrs}$ under general anesthesia were enrolled at the Zhongda Hospital and the Affiliated Hospital of Nanjing University of Traditional Chinese Medicine from September 2017 to October 2017. Exclusion criteria included bladder outflow obstruction, overactive bladder (frequency greater than three times per night or more than eight times per $24 \mathrm{~h}$ ), drug use for benign prostatic hyperplasia, history of urethral surgery, multisystemic diseases (cardiovascular, neuropsychiatric, hepatic, or renal dysfunction), chemical substance abuse, chronic pain or known allergy to medications used in the present trial.

\section{Randomization}

The patients were randomly allocated into one of the two groups (dezocine or control group) with the help of a computer-generated random number table. The assignments were concealed in opaque envelopes and opened by two anesthesiologists who administered the study drugs in the two hospitals. All outcomes were assessed by the other two anesthesiologists who were blinded to the group assignments.

\section{Study intervention}

During the preoperative visit, patients were told about the symptoms of CRBD. No preoperative medicine was used. After establishing intravenous access in the operating room, monitors for electrocardiogram, peripheral oxygen saturation, blood pressure and temperature were applied to all patients. Following preoxygenation with $100 \%$ oxygen, anesthesia was induced with midazolam $0.04 \mathrm{mg} / \mathrm{kg}$, sufentanil $0.3 \mu \mathrm{g} / \mathrm{kg}$ and propofol $1.5-2.5 \mathrm{mg} / \mathrm{kg}$. Endotracheal intubation was facilitated by rocuronium $0.6 \mathrm{mg} / \mathrm{kg}$. Ventilation was mechanically controlled to maintain the end tidal carbon dioxide tension at $35-40 \mathrm{mmHg}$. Then, urinary catheterization was performed with a 14 or $16 \mathrm{Fr}$ Foley catheter, and its balloon was inflated with $10 \mathrm{ml}$ saline. The catheter was lubricated with paraffin oil before insertion and was fixed to the leg with adhesive tape without traction after successful insertion. Patients with complicated catheter insertion requiring more than 3 repeated attempts were dropped from the present trial. Anesthesia was maintained using 2\%-3\% sevoflurane in a mixture of $50 \%$ oxygen and $50 \%$ air, with intermittent intravenous injection of sufentanil and cis-atracurium as needed. Patients received intravenous dezocine $0.1 \mathrm{mg} / \mathrm{kg}$ (dezocine group, $\mathrm{n}=48$ ) or flurbiprofen axetil $1 \mathrm{mg} / \mathrm{kg}$ (control group, $\mathrm{n}=48$ ) $20-30$ mins before the end of surgery according to the assignment. After the end of surgery, ondansetron $8 \mathrm{mg}$ was used as a prophylaxis for postoperative nausea and vomiting, and patients were transferred to the post anesthesia care unit. When patients regained adequate spontaneous ventilation and responded to commands, the tracheal tube was extubated. Then, patients received patient-controlled analgesia with sufentanil $(0.0125 \mu \mathrm{g} / \mathrm{kg}$ bolus with a 15 min lock-out and $0.04 \mu \mathrm{g} / \mathrm{kg} / \mathrm{h}$ background infusion for laparotomy and $0.02 \mu \mathrm{g} / \mathrm{kg} / \mathrm{h}$ for laparoscopy 2 days)

\section{Outcomes}

The primary outcome was the incidence of CRBD at $1 \mathrm{hr}$ after tracheal extubation. The secondary outcome measures included the incidence and severity of CRBD, numeric rating scale (NRS), Ramsay sedation score, and adverse effects (postoperative nausea and vomiting, dizziness, respiratory 
depression, oversedation and hypotension/hypertension) at 0 , 1,2 and $6 \mathrm{hrs}$ after extubation. The overall incidence and overall severity from 0 to $6 \mathrm{hrs}$ after extubation were also calculated. The overall incidence was defined as the ratio of patients who experienced CRBD at any time point to total patients and the overall severity was analyzed according to the highest level of CRBD severity that patients experienced within $6 \mathrm{hrs}$ after extubation. The severity of CRBD was recorded as: mild (reported by the patient only on questioning), moderate (reported by the patient on their own but without any behavioral response) and severe (reported by the patient on their own along with behavioral responses, including flailing limbs, vocal responses and attempts to pull out catheter). ${ }^{9-23}$ When severe CRBD occurred, a rescue therapy of $1 \mathrm{mg} / \mathrm{kg}$ intravenous tramadol was administered. Postoperative pain was assessed using a verbal NRS (0 represents "no pain" and 10 represents "the worst pain possible") Fentanyl $50 \mu \mathrm{g}$ was supplemented as a rescue therapy in cases with an NRS $\geq 4$, and cases were removed from the present trial to avoid the confounding analgesic effect of fentanyl on the development of CRBD. The Ramsay sedation score was evaluated at the same time points and recorded as follows: 1 (anxious, agitated or restless); 2 (cooperative, oriented and tranquil); 3 (responds to commands, asleep); 4 (brisk response to light glabellar tap or loud noise); 5 (sluggish response to light glabellar taps or loud noise) or 6 (no response). The incidences of postoperative nausea and vomiting, dizziness, respiratory depression (respiratory rate $<8$ breaths per minute, or $\mathrm{SpO}_{2}<90 \%$ ), oversedation (Ramsay score $\geq 5$ ), hypotension (systolic blood pressure $<90 \mathrm{mmHg}$ or $30 \%$ lower than the pretreatment value) and hypertension (systolic blood pressure $>180 \mathrm{mmHg}$ or $30 \%$ higher than the pretreatment value) were also recorded.

\section{Statistical analyses}

The sample size was calculated by PASS 15.0 (NCSS, Utah, USA). According to previous studies, approximately $70 \%$ of patients complained of CRBD postoperatively. ${ }^{3,30}$ Assuming that dezocine reduces the incidence of CRBD by $30 \%, 38$ patients are needed in each group to detect statistical significance with $\alpha=0.05$ and $\beta=0.10$. Considering a $20 \%$ dropout rate, 48 patients were included per group in the present trial. SPSS 17.0 (SPSS Inc., Chicago, IL, USA) was used for statistical analysis. Patient characteristics were compared by independent samples $t$-test for continuous variables and chi-squared test/Mann-Whitney $U$ test for categorical variables. The incidence of CRBD, postoperative nausea and vomiting, dizziness and the number of patients receiving tramadol or fentanyl treatment were compared by the chi-squared test or Fisher's exact test. The severity of CRBD was compared by the Mann-Whitney $\mathrm{U}$ test. The NRS and Ramsay scores were analyzed by repeated measures analysis of variance, followed by Tukey's test for post hoc comparisons. $P<0.05$ was considered statistically significant. Data are expressed as the mean (SD) or the number (proportion) as appropriate.

\section{Results}

A total of 121 patients were screened from September to October 2017 for inclusion in the present trial (Figure 1). Twenty-five patients were excluded [exclusion criteria $(n=15)$, refusal $(n=7)$ and cancellation of surgery $(n=3)]$. Thus, 96 patients $(n=48$ in the dezocine and control groups, respectively) were enrolled and all patients completed the present trial. There were no significant differences in the demographic data between the two groups (Table 1).

The primary outcome showed a lower incidence of CRBD at $1 \mathrm{hr}$ post-extubation in the dezocine group $(29.17 \%)$ than the control group $(58.33 \%)(P<0.01)$. The secondary outcome showed that the incidence of CRBD was lower in the dezocine group than in the control group at $0[(20.83 \%)$ vs $(58.33 \%) ; P=0.000]$, and $2 \mathrm{hrs}$ $[(31.25 \%)$ vs $(52.08 \%) ; \quad P=0.038]$ but not 6 hrs [(20.83\%) vs $(33.33 \%) ; P=0.168]$ after tracheal extubation. However, the severity of CRBD was comparable at $0,1,2$ and 6 hrs after extubation between the two groups $(P>0.05)$. The overall incidence of CRBD was lower in the dezocine group than in the control group [(41.67\%) vs (66.67\%); $P=0.014]$. The overall severity of CRBD was lower in the dezocine group compared with the control group ( $P=0.048$ ) (Table 2$)$. The numbers of patients treated with tramadol for CRBD were $1(2.08 \%)$ and 3 $(6.25 \%)$ in the dezocine and control groups, respectively $(P=0.617)$.

No patient needed rescue analgesia with fentanyl for postoperative pain relief. The NRS at $0,1,2$ and $6 \mathrm{hrs}$ after extubation were comparable between the two groups $(P>0.05)$. The Ramsay sedation scores at $0,1,2$ and 6 hrs after extubation were comparable between the two groups $(P>0.05)$. (Table 3$)$

No significant difference was observed in the overall incidence of postoperative nausea and vomiting and dizziness between the dezocine and control groups within $6 \mathrm{hrs}$ after extubation $(P>0.05)$. No patient experienced respiratory depression, oversedation or hypotension/hypertension in the present trial. (Table 4) 


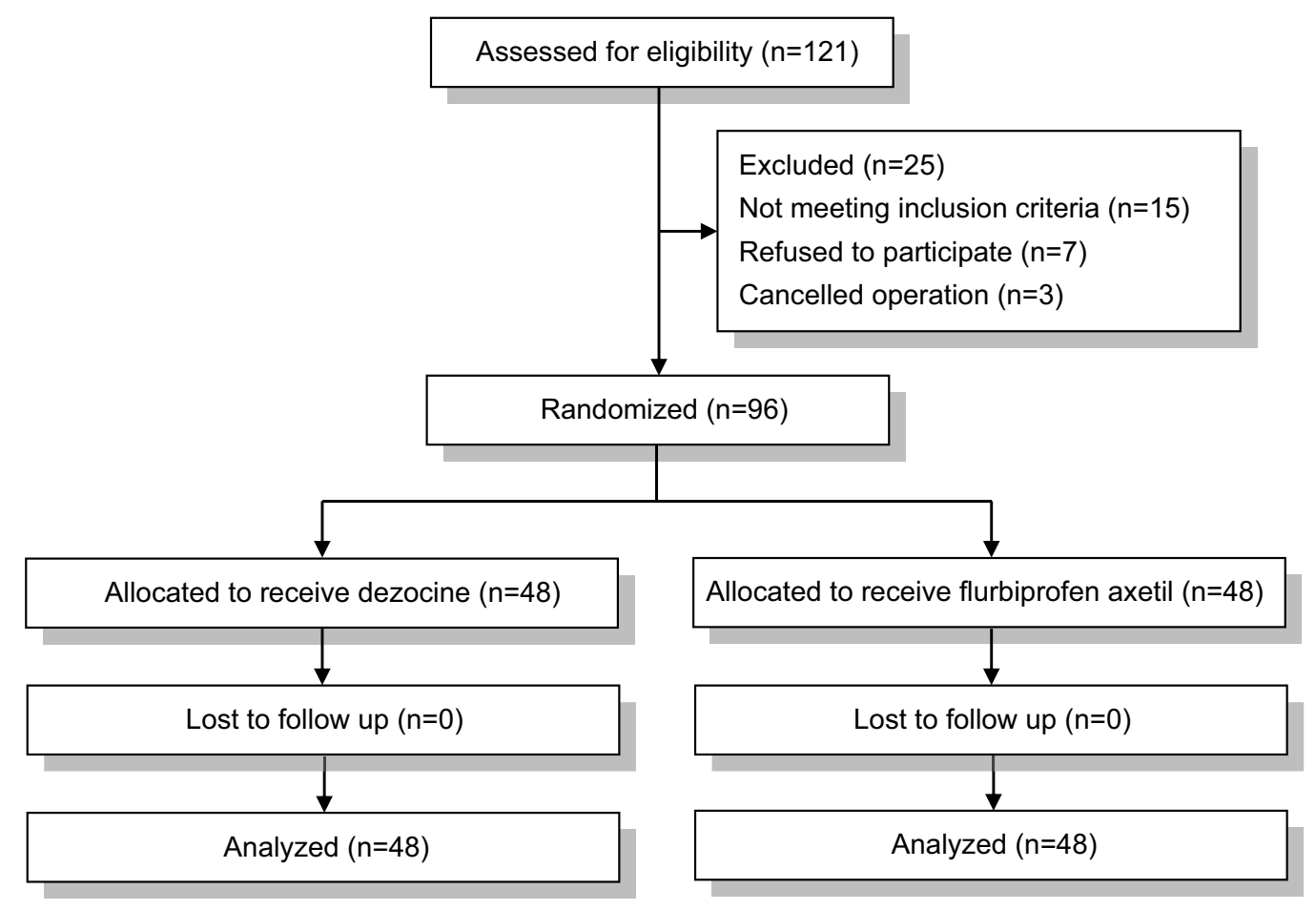

Figure I Flowchart of study progress.

\section{Discussion}

The present trial demonstrated that dezocine administration before the end of the surgery reduced the incidence and severity of postoperative CRBD without inducing severe drug-related adverse effects.

CRBD, a high incidence complication during the postoperative period, deserves more attention from anesthesiologists and surgeons. Although many pharmacologic therapies have been applied in the prevention or treatment of CRBD, the adverse effects of the majority of drugs have limited their clinical application. ${ }^{9-23}$

Dezocine, an opioid medication, was approved by the Food and Drug Administration for perioperative pain management after its development in the 1970s but was discontinued with the closure of its parent company. ${ }^{26}$ Recently, dezocine has been commonly used in China. Dezocine interacts with three major opioid receptors, ie, $\mu, \kappa$ and $\delta$, and the unique molecular pharmacological profile of dezocine as a partial $\mu$ receptor agonist reduces its adverse reactions in comparison with other pure opioids. Dezocine has been proven to be effective in relieving moderate to severe surgical pain, ${ }^{27-29}$ which was also shown in the present trial. CRBD is usually resistant to conventional opioids, however, the activation of $\kappa$ receptors by dezocine may contribute to the improvement of CRBD through inhibiting nociceptive stimulation from spasms of the vesical neck and urethral mucosal injury. In addition, dezocine did not increase the overall incidence of postoperative nausea and vomiting, dizziness, respiratory depression, oversedation or hypotension/ hypertension.

Previous studies investigated the incidence and severity of CRBD at 0, 1, 2, 6 and $24 \mathrm{hrs}$ postoperatively. ${ }^{3,10,17,31} \mathrm{In}$ this trial, since the elimination half-life of dezocine ranges from 1.2 to $7.4 \mathrm{hrs}$ and the catheters were removed in most patients within $24 \mathrm{hrs}$ postoperatively, we selected the time points of $0,1,2$ and $6 \mathrm{hrs}$ after tracheal extubation. All patients were resting in bed for at least $6 \mathrm{hrs}$ after tracheal extubation in the present study. Dezocine reduced the incidence of CRBD at 0,1 and 2 hrs but not 6 hrs after tracheal extubation. The overall severity of CRBD was attenuated by dezocine but not the severity at 0,1,2 and $6 \mathrm{hr}$ assessments, probably due to the small sample size calculated based on differences in incidence rather than in severity.

Previous studies showed that sevoflurane, as a maintenance agent of general anesthesia, reduced the incidence of CRBD during the first $24 \mathrm{hrs}$ postoperatively in patients undergoing transurethral resection of bladder tumors when compared with desflurane (76\% vs $93 \%)^{30}$ and propofol (66\% vs $\left.93 \%\right){ }^{3}$ Dexmedetomidine administration during surgery decreased the incidence of 
Table I Characteristics of study population

\begin{tabular}{|c|c|c|c|}
\hline & Dezocine $(n=48)$ & Control $(n=48)$ & $P$-value \\
\hline Age (years) & $53.19(11.99)$ & $52.56(11.67)$ & 0.796 \\
\hline Sex (male/female) & $36 / 12$ & $34 / 14$ & 0.646 \\
\hline Weight (kg) & $69.73(11.72)$ & $67.81(12.20)$ & 0.434 \\
\hline Height $(\mathrm{cm})$ & $168.88(6.92)$ & $166.93(6.86)$ & 0.171 \\
\hline BMI $\left(\mathrm{kg} \cdot \mathrm{m}^{-2}\right)$ & $24.42(3.74)$ & $24.20(3.22)$ & 0.757 \\
\hline ASA physical status (I/II) & $28 / 20$ & $25 / 23$ & 0.538 \\
\hline Duration of surgery $(\mathrm{min})$ & $99.17(56.57)$ & $108(71.11)$ & 0.225 \\
\hline Duration of anesthesia (min) & $122.02(60.55)$ & $137.40(75.29)$ & 0.273 \\
\hline Time to tracheal extubation (min) & $30.10(14.20)$ & $33.54(14.77)$ & 0.248 \\
\hline Urinary catheter size & & & 0.186 \\
\hline $14 \mathrm{Fr}$ & $12(25.00)$ & $18(37.50)$ & \\
\hline $16 \mathrm{Fr}$ & $36(75.00)$ & $30(62.50)$ & \\
\hline Type of operation & & & 0.332 \\
\hline Stomach & $6(12.50)$ & $10(20.83)$ & \\
\hline Colorectal & $8(16.67)$ & $9(18.75)$ & \\
\hline Gallbladder & $18(37.50)$ & $15(31.25)$ & \\
\hline Hernia & $16(33.33)$ & $14(29.17)$ & \\
\hline Operative approach & & & 0.283 \\
\hline Laparotomy & $14(29.17)$ & $19(39.58)$ & \\
\hline Laparoscopic & $34(70.83)$ & $29(60.42)$ & \\
\hline \multicolumn{4}{|l|}{ Intraoperative fluid volume } \\
\hline Lactated ringer's solution & II $66.67(429.41)$ & $1083.33(465.09)$ & 0.36 \\
\hline $6 \%$ hydroxyethyl starch & $319.79(218.98)$ & $355.83(204.53)$ & 0.41 \\
\hline Intraoperative urine volume & $312.92(183.01)$ & $354.17(195.91)$ & 0.29 \\
\hline Intraoperative sufentanil requirement $(\mu \mathrm{g})$ & $53.13(17.7 I)$ & $56.51(16.01)$ & 0.33 \\
\hline Postoperative sufentanil requirement within $6 \mathrm{hrs}(\mu \mathrm{g})$ & $11.13(4.92)$ & $12.10(5.48)$ & 0.36 \\
\hline
\end{tabular}

Note: Data are presented as the mean (SD) or the number (\%) of patients.

CRBD in patients undergoing TURBT (57\% vs $85 \%)^{17}$ or elective open abdominal surgery $(20 \% \text { vs } 68 \%)^{31}$ when compared with the control group at $1 \mathrm{hr}$ postoperatively. Reductions in the CRBD incidence ranging from $23 \%$ to $50 \%$ following anticholinergic and antiepileptic administration have been reported in previous studies. $^{5,10,19}$ In the present trial, the reduction in CRBD incidence at $1 \mathrm{hr}$ after extubation was $29.16 \%$ in the dezocine group compared with the control group. These results suggested that the application of anticholinergics, sevoflurane (rather than desflurane or propofol), or dezocine (rather than flurbiprofen) during the anesthesia procedure may be beneficial for preventing postoperative CRBD. Multiple drugs, such as sevoflurane and midazolam, which may help with CRBD were used in both groups in the present trial; therefore, the effects of these drugs on CRBD could not be accounted for in the study design. The use of the PCA was matched in the two groups and there was no significant difference in the overall sufentanil dose postoperatively.

Saline served as a control for the prevention of CRBD in previous studies and may increase patients' pain experience. ${ }^{14,15,17}$ Flurbiprofen axetil, a nonsteroid antiinflammatory drug, was used for postoperative multimodal analgesia and as a control in the present trial. Although Ergenoglu et al. ${ }^{23}$ reported that paracetamol, a COX-2 inhibitor, alleviated the occurrence and symptoms of CRBD, flurbiprofen axetil seemed to be ineffective for CRBD; the incidence of CRBD in the flurbiprofen axetil control group $58.33 \%$ in all patients and $67.65 \%$ in male patients) were similar to the saline controls in previous studies ( $58 \%$ in all patients ${ }^{10}$ and $68 \%$ in male patients ${ }^{31}$ ).

The study was initially registered at clinicaltrials.gov with a placebo control arm (NCT03147066), which was changed to flurbiprofen control. This change was made because of a suggestion from the Institutional Ethics 
Table 2 Incidence and severity of postoperative catheter-related bladder discomfort

\begin{tabular}{|c|c|c|c|}
\hline & Dezocine $(n=48)$ & Control $(n=48)$ & $P$-value \\
\hline $\begin{array}{l}\text { Postextubation } 0 \mathrm{~h} \\
\text { Incidence }\end{array}$ & $10(20.83)$ & $28(58.33)$ & 0.000 \\
\hline $\begin{array}{l}\text { Severity } \\
\text { Mild } \\
\text { Moderate } \\
\text { Severe }\end{array}$ & $\begin{array}{l}6(12.50) \\
3(6.25) \\
1(2.08)\end{array}$ & $\begin{array}{l}14(29.17) \\
12(25.00) \\
2(4.17)\end{array}$ & 0.682 \\
\hline $\begin{array}{l}\text { Postextubation I hrs } \\
\text { Incidence }\end{array}$ & $14(29.17)$ & $28(58.33)$ & 0.004 \\
\hline $\begin{array}{l}\text { Severity } \\
\text { Mild } \\
\text { Moderate } \\
\text { Severe }\end{array}$ & $\begin{array}{l}\text { I3 (27.08) } \\
\text { I (2.08) } \\
0(0)\end{array}$ & $\begin{array}{l}19(39.58) \\
8(16.67) \\
1(2.08)\end{array}$ & 0.189 \\
\hline $\begin{array}{l}\text { Postextubation } 2 \text { hrs } \\
\text { Incidence }\end{array}$ & $15(31.25)$ & $25(52.08)$ & 0.038 \\
\hline $\begin{array}{l}\text { Severity } \\
\text { Mild } \\
\text { Moderate } \\
\text { Severe }\end{array}$ & $\begin{array}{l}12(25.00) \\
3(6.25) \\
0(0)\end{array}$ & $\begin{array}{l}17(35.42) \\
8(16.67) \\
0(0)\end{array}$ & 0.416 \\
\hline $\begin{array}{l}\text { Postextubation } 6 \text { hrs } \\
\text { Incidence }\end{array}$ & $10(20.83)$ & $16(33.33)$ & 0.168 \\
\hline $\begin{array}{l}\text { Severity } \\
\text { Mild } \\
\text { Moderate } \\
\text { Severe }\end{array}$ & $\begin{array}{l}10(20.83) \\
0(0) \\
0(0)\end{array}$ & $\begin{array}{l}16(33.33) \\
0(0) \\
0(0)\end{array}$ & 1.000 \\
\hline $\begin{array}{l}\text { Overall } \\
\text { Incidence }\end{array}$ & $20(4 \mid .67)$ & $32(66.67)$ & 0.014 \\
\hline $\begin{array}{l}\text { Severity } \\
\text { Mild } \\
\text { Moderate } \\
\text { Severe }\end{array}$ & $\begin{array}{l}14(29.17) \\
5(10.42) \\
1(2.08)\end{array}$ & $\begin{array}{l}13(27.08) \\
16(33.33) \\
3(6.25)\end{array}$ & 0.048 \\
\hline
\end{tabular}

Notes: Data are presented as the number (\%) of patients. CRBD, catheter-related bladder discomfort.

Committee for ruling out the analgesic effect of dezocine on the occurrence of CRBD. A few limitations of the present trial should be considered. First, we did not completely rule out confounding factors such as the sedative effect of dezocine on the incidence of CRBD. Although the Ramsay sedation score was comparable between the two groups, we could not exclude the possibility that the sedative effect of dezocine did not mask symptoms of CRBD. Second, we could not blind the two anesthesiologists who administered the drugs because of the different drug appearances. Thus, the other two anesthesiologists blinded to the study drugs performed the outcome measurements. Third, it remains uncertain whether flurbiprofen axetil if of benefit for CRBD because no saline controls were included in the present trial. Fourth, although no significant difference between groups was observed in the intraoperative fluid volume and urine volume, the effects of the postoperative volume of fluid and urine on CRBD should not be completely neglected in the present trial.

\section{Conclusion}

Dezocine administration before the end of surgery attenuated the incidence and severity of CRBD without inducing severe adverse effects for patients undergoing 
Table 3 Postoperative pain and sedation scores

\begin{tabular}{|c|c|c|c|c|c|c|c|c|}
\hline \multirow{2}{*}{$\begin{array}{l}\text { Time (hrs) } \\
\text { Groups } \\
(n=48)\end{array}$} & \multicolumn{2}{|l|}{0} & \multicolumn{2}{|l|}{ I } & \multicolumn{2}{|l|}{2} & \multicolumn{2}{|l|}{6} \\
\hline & Dezocine & Control & Dezocine & Control & Dezocine & Control & Dezocine & Control \\
\hline NRS & 0.85 & 0.96 & 1.06 & 1.06 & 0.85 & 0.85 & 0.85 & 0.83 \\
\hline$(0-10)$ & $(0.62-1.09)$ & $(0.69-1.22)$ & $(0.84-1.28)$ & $(0.78-1.35)$ & $(0.63-1.08)$ & $(0.62-1.09)$ & $(0.62-1.09)$ & $(0.62-1.04)$ \\
\hline Ramsay score & 2.04 & 1.98 & 1.98 & 1.96 & 2.02 & 1.94 & 1.94 & 1.96 \\
\hline$(I-6)$ & $(1.94-2.14)$ & $(1.91-2.05)$ & $(1.93-2.02)$ & $(1.90-2.02)$ & $(1.95-2.10)$ & $(1.87-2.01)$ & $(1.87-2.01)$ & $(1.90-2.02)$ \\
\hline
\end{tabular}

Note: Data are presented as the mean ( $95 \%$ confidence interval for mean).

Table 4 Overall incidence of adverse effects

\begin{tabular}{|l|l|l|l|}
\hline & $\begin{array}{l}\text { Dezocine } \\
(\mathbf{n = 4 8 )}\end{array}$ & $\begin{array}{l}\text { Control } \\
(\mathbf{n = 4 8 )}\end{array}$ & P-value \\
\hline Nausea/vomiting & $8(16.67)$ & $3(6.25)$ & 0.109 \\
Dizziness & $3(6.25)$ & $\mathrm{I}(2.08)$ & 0.617 \\
Respiratory depression & $0(0)$ & $0(0)$ & \\
Oversedation & $0(0)$ & $0(0)$ & \\
Hypotension/Hypertension & $0(0)$ & $0(0)$ & \\
\hline
\end{tabular}

Note: Data are presented as absolute frequency (\%). No additional data are available.

elective abdominal surgery with general anesthesia. These results suggest the application of dezocine as an analgesic for postoperative pain in patients at high risk of CRBD.

\section{Disclosure}

The authors report no conflicts of interest in this work.

\section{References}

1. Agarwal A, Raza M, Singhal V, et al. The efficacy of tolterodine for prevention of catheter-related bladder discomfort: a prospective, randomized, placebo-controlled, double-blind study. Anesth Analg. 2005;101(4):1065-1067. doi:10.1213/01.ane.0000167775.46192.e9

2. Bai Y, Wang X, Li X, et al. Management of catheter-related bladder discomfort in patients who underwent elective surgery. $J$ Endourol. 2015;29(6):640-649. doi:10.1089/end.2014.0670

3. Kim HC, Park HP, Lee J, Jeong M-H, Lee K-H. Sevoflurane vs. propofol in post-operative catheter-related bladder discomfort: a prospective randomized study. Acta Anaesthesiol Scand. 2017;61 (7):773-780. doi:10.1111/aas.12922

4. Hu B, Li C, Pan M, et al. Strategies for the prevention of catheter-related bladder discomfort: a PRISMA-compliant systematic review and meta-analysis of randomized controlled trials. Medicine (Baltimore). 2016;95(37):e4859. doi:10.1097/MD.0000000000004864

5. Kim JA, Min JH, Lee HS, Jo HR, Je UJ, Paek JH. Effects of glycopyrrolate premedication on preventing postoperative catheter-related bladderdiscomfort in patients receiving ureteroscopic removal of ureter stone. Korean J Anesthesiol. 2016;69:563-567. doi:10.4097/ kjae.2016.69.6.563

6. Tauzin-Fin P, Sesay M, Svartz L, Krol-Houdek M-C, Maurette P. Sublingual oxybutynin reduces postoperative pain related to indwelling bladder catheter after radical retropubic prostatectomy. $\mathrm{Br}$ J Anaesth. 2007;99(4):572-575. doi:10.1093/bja/aem232
7. Guenther U, Radtke FM. Delirium in the postanaesthesia period. Curr Opin Anaesthesiol. 2011;24(6):670-675. doi:10.1097/ ACO.0b013e $32834 \mathrm{c} 7 \mathrm{~b} 44$

8. Lepouse C, Lautner CA, Liu L, Gomis P, Leon A. Emergence delirium in adults in the postanaesthesia care unit. $\mathrm{Br} J$ Anaesth. 2006;96(6):747. doi:10.1093/bja/ael094

9. Ryu JH, Hwang JW, Lee JW, et al. Efficacy of butylscopolamine for the treatment of catheter-related bladder discomfort: a prospective, randomized, placebo-controlled, double-blind study. Br J Anaesth. 2013;111(6):932-937. doi:10.1093/bja/aet249

10. Agarwal A, Dhiraaj S, Singhal V, Kapoor R, Tandon M. Comparison of efficacy of oxybutynin and tolterodine for prevention of catheter related bladder discomfort: a prospective, randomized, placebo-controlled, double-blind study. Br J Anaesth. 2006;96(3):377-380. doi:10.1093/ bja/ael003

11. Chung J, Ha H, Kim D, et al. Evaluation of the efficacy of solifenacin for preventing catheter-related bladder discomfort after transurethral resection of bladder tumors in patients with non-muscle invasive bladder cancer: a prospective, randomized, multicenter study. Clin Genitourin Cancer. 2017;15(1):157-162. doi:10.1016/j.clgc.2016.05.006

12. Srivastava V, Nigam R, Agrawal S, Kumar S, Rambhad S, Kanaskar J. Evaluation of the efficacy of solifenacin and darifenacin for prevention of catheter-related bladder discomfort: a prospective, randomized, placebo-controlled, double-blind study. Minerva Anestesiol. 2016;82(8):867-873.

13. Kim H, Lim S, Seo H, Park H-P. Effect of glycopyrrolate versus atropine coadministered with neostigmine for reversal of rocuronium on postoperative catheter-related bladder discomfort in patients undergoing transurethral resection of bladder tumor: a prospective randomized study. J Anesth. 2015;29(6):831-835. doi:10.1007/ s00540-015-2064-2

14. Safavi M, Honarmand A, Atari M, Chehrodi S, Amoushahi M. An evaluation of the efficacy of different doses of ketamine for treatment of catheter-related bladder discomfort in patients underwent urologic surgery: a prospective, randomized, placebo-controlled, double-blind study. Urol Ann. 2014;6(1):51-56. doi:10.4103/09747796.127030

15. Agarwal A, Yadav G, Gupta D, Singh PK, Singh U. Evaluation of intra-operative tramadol for prevention of catheter-related bladder discomfort: a prospective, randomized, double-blind study. $\mathrm{Br}$ J Anaesth. 2008;101(4):506-510. doi:10.1093/bja/aen217

16. Kwon Y, Jang JS, Hwang SM, Lee JJ, Tark H. Intraoperative administration of dexmedetomidine reduced the postoperative catheter-relatedbladder discomfort and pain in patients undergoing lumbar microdiscectomy. $J$ Anesth. 2018;32(1):41-47. doi:10.1007/s00540-017-2425-0

17. Kim HC, Lee YH, Jeon YT, et al. The effect of intraoperative dexmedetomidine on postoperative catheter-related bladder discomfort in patients undergoing transurethral bladder tumour resection: a prospective, double-blind, randomised study. Eur J Anaesthesiol. 2015;32(9):596-601. doi:10.1097/EJA.0000000000000196 
18. Mu L, Geng L, Xu H, Luo M, Geng J-M, Li L. Lidocaineprilocaine cream reduces catheter-related bladder discomfort in male patients during the general anesthesia recovery period: a prospective, randomized, case-control STROBE study. Medicine (Baltimore). 2017;96(14):e6494. doi:10.1097/MD.0000000000 006494

19. Agarwal A, Dhiraaj S, Pawar S, Kapoor R, Gupta D, Singh PK. An evaluation of the efficacy of gabapentin for prevention of catheter-related bladder discomfort: a prospective, randomized, placebo-controlled, double-blind study. Anesth Analg. 2007;105(5):1454-1457. doi:10.1213/01.ane.0000281154.03 $887.2 \mathrm{~b}$

20. Srivastava VK, Agrawal S, Kadiyala VN, Ahmed M, Sharma S, Kumar R. The efficacy of pregabalin for prevention of catheter-related bladder discomfort: a prospective, randomized, placebo-controlled double-blind study. J Anesth. 2015;29 (2):212-216. doi:10.1007/s00540-014-1911-x

21. Verma R, Agarwal A, Singh PK, Gupta D, Shamim R. Evaluation of efficacy of amikacin for attenuation of catheter-related bladder discomfort in patients undergoing percutaneous nephrolithotomy: a prospective, randomized, placebo-controlled, double-blind study. Anesth Essays Res. 2016;10(3):613-617. doi:10.4103/02591162.191116

22. Zhang N, Zhang P, Zhang X, Yang Y. The efficacy of resiniferatoxin in prevention of catheter related bladder discomfort in patients after TURP-A pilot, randomized, open study. Transl Androl Urol. 2012;1 (1):14-18. doi:10.3978/j.issn.2223-4683.2012.01.02

23. Ergenoglu P, Akin S, Yalcin Cok O, et al. Effect of intraoperative paracetamol on catheter-related bladder discomfort: a prospective, randomized, double-blind study. Curr Ther Res Clin Exp. 2012;73 (6):186-194. doi:10.1016/j.curtheres.2012.08.001
24. Li J, Yi M, Liao R. Dorsal penile nerve block with ropivacaine-reduced postoperative catheter-related bladder discomfort in male patients after emergence of general anesthesia: a prospective, randomized, controlled study. Medicine (Baltimore). 2016;95(15):e3409. doi:10.1097/ MD.0000000000004864

25. Fragen RJ, Caldwell N. Comparison of dezocine (WY 16, 225) and meperidine as postoperative analgesics. Anesth Analg. 1978;57 (5):563-566.

26. Liu R, Huang XP, Yeliseev A, Xi J, Roth BL. Novel molecular targets of dezocineand their clinical implications. Anesthesiology. 2014;120 (3):714-723. doi:10.1097/ALN.0000000000000076

27. Yu F, Zhou J, Xia S, Xu H, Wang X. Dezocine prevents postoperative hyperalgesia in patients undergoing open abdominal surgery. Evid Based Complement Alternat Med. 2015;2015:946194. doi:10.1155/2015/946194

28. Fang BX, Wang LH, Liu HM, Chen F-C, Liu J. Stability study of dezocine in $0.9 \%$ sodium chloride solutions for patient-controlled analgesia administration. Medicine (Baltimore). 2017;96(35):e7979. doi:10.1097/MD.0000000000007979

29. Zhou X, Zhang C, Wang M, Yu L, Yan M, Gemma M. Dezocine for preventing postoperative pain: a meta-analysis of randomized controlled trials. PLoS One. 2015;10(8):e0136091. doi:10.1371/journal. pone. 0136091

30. Kim HC, Hong WP, Lim YJ, Park H-P. The effect of sevoflurane versus desflurane on postoperative catheter-related bladder discomfort in patients undergoing transurethral excision of a bladder tumour: a randomized controlled trial. Can J Anaesth. 2016;63(5):596-602. doi:10.1007/s12630-016-0600-7

31. Yang ZY, Xu JM, Zhu R, Ru Ping D, Lei L. Dexmedetomidine reduces catheter-related bladder discomfort: a prospective, randomized, placebo- controlled, double-blind study. $P R$ Health Sci J. 2016;35(4):191-196.

\section{Publish your work in this journal}

Drug Design, Development and Therapy is an international, peerreviewed open-access journal that spans the spectrum of drug design and development through to clinical applications. Clinical outcomes, patient safety, and programs for the development and effective, safe, and sustained use of medicines are a feature of the journal, which has also been accepted for indexing on PubMed Central. The manuscript management system is completely online and includes a very quick and fair peer-review system, which is all easy to use. Visit http://www. dovepress.com/testimonials.php to read real quotes from published authors. 